\title{
NOTE ON THE GROUND STATES OF TWO-COMPONENT BOSE-EINSTEIN CONDENSATES WITH AN INTERNAL ATOMIC JOSEPHSON JUNCTION
}

\author{
ZhongXue Lü AND ZuHAN LiU
}

\begin{abstract}
In this paper, we consider two-component Bose-Einstein condensates with an internal atomic Josephson junction in the general case, i.e., $0<p<\frac{2}{(d-2)^{+}}$. We prove existence and uniqueness results for the ground states, and obtain some properties of the ground states with large parameters.
\end{abstract}

\section{Introduction}

We consider the following two-components nonlinear Schrödinger equations $[5,8,9,10]$,

(1.1)

$$
\begin{aligned}
& \left\{\begin{aligned}
i \psi_{t}^{1}= & -\frac{1}{2} \Delta \psi^{1}+|x|^{2} \psi^{1}+\delta \psi^{1}+\left(\left.v_{11} \psi^{1}\right|^{2 p} \psi^{1}+v_{12}\left|\psi^{2}\right|^{p+1}\left|\psi^{1}\right|^{p-1} \psi^{1}\right)+\lambda \psi^{2} \\
& \text { in } \mathbb{R}^{d} \times \mathbb{R}, \\
i \psi_{t}^{2}= & -\frac{1}{2} \Delta \psi^{2}+|x|^{2} \psi^{2}+\left(v_{12}\left|\psi^{1}\right|^{p+1}\left|\psi^{2}\right|^{p-1} \psi^{2}+v_{22}\left|\psi^{2}\right|^{2 p} \psi^{2}\right)+\lambda \psi^{1} \\
& \text { in } \mathbb{R}^{d} \times \mathbb{R},
\end{aligned}\right. \\
& \qquad \psi^{1}(x, 0)=\varphi^{1}(x), \psi^{2}(x, 0)=\varphi^{2}(x),
\end{aligned}
$$

where $t$ is time, $x \in \mathbb{R}^{d}(d=1,2,3)$ is the Cartesian coordinate vector, $\psi^{j}(x, t)$ : $\mathbb{R}^{n} \times \mathbb{R} \rightarrow \mathbb{C}$ is the corresponding macroscopic wave function of the $j^{t h}(j=1,2)$ component, and $\psi_{0}^{1}(x), \psi_{0}^{2}(x)$ is the initial data. $\lambda$ is the effective Rabi frequency to realize the internal atomic Josephson junction by a Raman transition, $\delta$ is the detuning constant for the Raman transition, $0<p<\frac{2}{(d-2)^{+}}\left((d-2)^{+}=\right.$ $+\infty$ when $d=1,2$, and $(d-2)^{+}=1$ when $\left.d \geq 3\right), v_{i j}, i, j=1,2$ are coupling constants, $v_{12}=v_{21}$ are the s-wave scattering lengths between the first and the

Received July 11, 2011; Revised February 2, 2013.

2010 Mathematics Subject Classification. 35Q55, 49J45.

Key words and phrases. Bose-Einstein condensate, two-component, ground state, internal atomic Josephson junction.

This work is supported by the Natural Science Foundation of China (No.11071206, 11271166), NSF of Jiangsu Province (No. BK2010172), sponsored by Qing Lan Project, and the Priority Academic Program Development of Jiangsu Higher Education Institutions. 
second component (positive for repulsive interaction and negative for attractive interaction). This model has applications in many problems, especially in Bose-Einstein condensates. It is necessary to ensure that the wave function is properly normalized. Especially, we require

$$
\int_{\mathbb{R}^{d}}\left[\left|\psi^{1}\right|^{2}+\left|\psi^{2}\right|^{2}\right] d x=1
$$

The dimensionless CGPEs (1.1) conserves the total mass or normalization, i.e.,

$$
N\left(\psi^{1}, \psi^{2}\right):=\left\|\psi^{1}\right\|_{L^{2}}^{2}+\left\|\psi^{2}\right\|_{L^{2}}^{2}=1, \quad t \geq 0
$$

with

$$
\left\|\psi^{j}\right\|_{L^{2}}^{2}=\int_{\mathbb{R}^{d}}\left|\psi^{j}\right|^{2} d x, \quad t \geq 0, j=1,2
$$

and the energy

$$
E\left(\psi^{1}, \psi^{2}\right)=E_{0}\left(\psi^{1}, \psi^{2}\right)+2 \lambda \int_{\mathbb{R}^{d}} \operatorname{Re}\left(\psi^{1} \overline{\psi^{2}}\right) d x,
$$

with $\bar{f}$ and $\operatorname{Re}(f)$ denoting the conjugate and real part of a function $f$, respectively, and

$$
\begin{aligned}
E_{0}\left(\psi^{1}, \psi^{2}\right):= & \int_{\mathbb{R}^{d}}\left[\frac{1}{2}\left(\left|\nabla \psi^{1}\right|^{2}+\left|\nabla \psi^{2}\right|^{2}\right)+|x|^{2}\left(\left|\psi^{1}\right|^{2}+\left|\psi^{2}\right|^{2}\right)+\delta\left|\psi^{1}\right|^{2}\right] d x \\
& +\frac{1}{p+1} \int_{\mathbb{R}^{n}}\left(v_{11}\left|\psi^{1}\right|^{2 p+2}+v_{22}\left|\psi^{2}\right|^{2 p+2}+2 v_{12}\left|\psi^{1}\right|^{p+1}\left|\psi^{2}\right|^{p+1}\right) d x .
\end{aligned}
$$

The ground state $\psi_{g}^{1}(x), \psi_{g}^{2}(x)$ of the two-component BEC with an internal atomic Josephson junction (1.1) is defined as:

If $\psi_{g}^{1}(x), \psi_{g}^{2}(x) \in S$ satisfies

$$
E_{g}:=E\left(\psi_{g}^{1}(x), \psi_{g}^{2}(x)\right)=\min _{\psi^{1}, \psi^{2} \in S} E\left(\psi^{1}(x), \psi^{2}(x)\right),
$$

where $S$ is a nonconvex set defined as

$$
S:=\left\{\phi^{1}(x), \phi^{2}(x):\left\|\phi^{1}\right\|_{L^{2}}^{2}+\left\|\phi^{2}\right\|_{L^{2}}^{2}=1, E\left(\phi^{1}(x), \phi^{2}(x)\right)<\infty\right\} .
$$

It is easy to see the ground state $\phi_{g}^{1}(x), \phi_{g}^{2}(x)$ satisfies the following EulerLagrange equations,

(1.4)

$\left\{\begin{array}{l}\mu \phi^{1}=-\frac{1}{2} \Delta \phi^{1}+|x|^{2} \phi^{1}+\delta \phi^{1}+\left(\left.v_{11} \phi^{1}\right|^{2 p} \phi^{1}+v_{12}\left|\phi^{2}\right|^{p+1}\left|\phi^{1}\right|^{p-1} \phi^{1}\right)+\lambda \phi^{2}, \\ \mu \phi^{2}=-\frac{1}{2} \Delta \phi^{2}+|x|^{2} \phi^{2}+\left(\left.v_{12} \phi^{1}\right|^{2 p} \phi^{2}+v_{22}\left|\phi^{2}\right|^{p+1}\left|\phi^{1}\right|^{p-1} \phi^{1}\right)+\lambda \phi^{1}, x \in \mathbb{R}^{d},\end{array}\right.$

under the constraint

$$
\left\|\phi^{1}\right\|_{L^{2}}^{2}+\left\|\phi^{2}\right\|_{L^{2}}^{2}=1 .
$$

In fact, the above time-independent CGPEs (1.4) can be obtained from the CGPEs (1.1) by substituting the ansatz

$$
\psi^{1}(x, t)=e^{-i \mu t} \phi^{1}(x), \psi^{2}(x, t)=e^{-i \mu t} \phi^{2}(x) .
$$


The motivation to study problem (1.4) comes from many interest concerning supercritical problems. There are some analytical studies for the ground states of two-component BEC without the internal atomic Josephson junction in the literatures $[1,3,4,7]$. For the ground states of two-component BEC with an internal atomic Josephson junction, Bao [2] established existence and uniqueness results as $p=1$ in (1.1). This is the critical case in $d=2$. To our knowledge, there are no analytical and numerical results for the ground states of two-component BEC with an internal atomic Josephson junction in the general case, i.e., $0<p<\frac{2}{(d-2)^{+}}$. The main aim of this paper is to establish existence and uniqueness results and some properties for the ground states of two-component BEC with an internal atomic Josephson junction in the general case, i.e., $0<p<\frac{2}{(d-2)^{+}}$.

The paper is organized as follows. In Section 2, we prove existence and uniqueness results for ground states. In Section 3, some properties of the ground states are established.

\section{Existence and uniqueness results for the ground states}

Let

$$
V=\left(\begin{array}{ll}
v_{11} & v_{12} \\
v_{21} & v_{22}
\end{array}\right)
$$

We say matrix $V$ is positive semi-definite if and only if $v_{11} \geq 0$ and $v_{11} v_{22}-$ $v_{12}^{2} \geq 0$; and $V$ is nonnegative if and only if $v_{11} \geq 0$ and $v_{12} \geq 0$ and $v_{22} \geq 0$.

Denote

$$
\mathcal{D}=\left\{\phi^{1}, \phi^{2}:\left|x \phi^{j}\right|^{2} \in L^{1}\left(\mathbb{R}^{d}\right), \phi^{j} \in H^{1}\left(\mathbb{R}^{d}\right) \cap L^{2 p+2}\left(\mathbb{R}^{d}\right), j=1,2\right\},
$$

then the ground state $\phi_{g}^{1}, \phi_{g}^{2}$ of (1.3) is also defined as:

If $\phi_{g}^{1}, \phi_{g}^{2} \in \mathcal{D}_{1}$ satisfies

$$
E\left(\phi_{g}^{1}, \phi_{g}^{2}\right)=\min _{\left.\phi^{1}, \phi^{2} \in \mathcal{D}_{1}\right)} E\left(\phi^{1}, \phi^{2}\right),
$$

where

$$
\mathcal{D}_{1}=\mathcal{D} \cap\left\{\phi^{1}, \phi^{2}:\left\|\phi^{1}\right\|_{L^{2}}^{2}+\left\|\phi^{2}\right\|_{L^{2}}^{2}=1\right\} .
$$

In addition, we introduce the auxiliary energy functional

$$
\widetilde{E}\left(\psi^{1}(x), \psi^{2}(x)\right)=E_{0}\left(\psi^{1}(x), \psi^{2}(x)\right)-2 \lambda \int_{\mathbb{R}^{d}}\left|\psi^{1}\right| \cdot\left|\psi^{2}\right| d x,
$$

and the auxiliary nonconvex minimization problem is as follows:

Find $\phi_{g}^{1}, \phi_{g}^{2} \in \mathcal{D}_{1}$, such that

$$
\widetilde{E}\left(\phi_{g}^{1}, \phi_{g}^{2}\right)=\min _{\phi^{1}, \phi^{2} \in \mathcal{D}_{1}} \widetilde{E}\left(\phi^{1}, \phi^{2}\right) .
$$

Similarly to [2], we have the following lemmas: 
Lemma 2.1. For the minimizers $\phi_{g}^{1}, \phi_{g}^{2}$ of the nonconvex minimization problems (2.1) and (2.3), we have

i) If $\phi_{g}^{1}, \phi_{g}^{2}$ is a minimizer of (2.1), then $\phi_{g}^{1}(x)=e^{i \theta_{1}}\left|\phi_{g}^{1}(x)\right|$ and $\phi_{g}^{2}(x)=$ $e^{i \theta_{2}}\left|\phi_{g}^{2}(x)\right|$ with $\theta_{1}$ and $\theta_{2}$ two constants satisfying $\theta_{1}=\theta_{2}$ if $\lambda<0$; and $\theta_{1}=\theta_{2} \pm \pi$ if $\lambda>0$. In addition, $e^{i \theta_{3}} \phi_{g}^{1}, e^{i \theta_{4}} \phi_{g}^{2}$ with $\theta_{3}$ and $\theta_{4}$ two constants satisfying $\theta_{3}=\theta_{4}$ if $\lambda<0$; and $\theta_{3}=\theta_{4} \pm \pi$ if $\lambda>0$ is also a minimizer of $(2.3)$.

ii) If $\phi_{g}^{1}, \phi_{g}^{2}$ is a minimizer of (2.3), then $\phi_{g}^{1}(x)=e^{i \theta_{1}}\left|\phi_{g}^{1}(x)\right|$ and $\phi_{g}^{2}(x)=$ $e^{i \theta_{2}}\left|\phi_{g}^{2}(x)\right|$ with $\theta_{1}$ and $\theta_{2}$ two constants. In addition, $e^{i \theta_{3}} \phi_{g}^{1}, e^{i \theta_{4}} \phi_{g}^{2}$ with $\theta_{3}$ and $\theta_{4}$ two constants is also a minimizer of (2.3).

iii) If $\phi_{g}^{1}, \phi_{g}^{2}$ is a minimizer of (2.1), then $\phi_{g}^{1}, \phi_{g}^{2}$ is also a minimizer of (2.3).

iv) If $\phi_{g}^{1}, \phi_{g}^{2}$ is a minimizer of (2.3), then $\left|\phi_{g}^{1}\right|,-\operatorname{sign}(\lambda)\left|\phi_{g}^{2}\right|$ is a minimizer of $(2.2)$.

Lemma 2.2. Assume that $v_{11} \geq 0,(p-1) v_{12} \geq 0$ and $v_{11} v_{22} \geq \max \left\{\frac{1}{p}, 1\right\} v_{12}^{2}$ and at least one of the parameters $\lambda, \gamma_{1}=v_{11}-v_{22}$ and $\gamma_{2}=v_{11}-v_{12}$ is nonzero for $\rho_{1}, \rho_{2}$ with $\rho_{1}, \rho_{2} \geq 0, \sqrt{\rho_{1}}, \sqrt{\rho_{2}} \in \mathcal{D}_{1}$. Then $\widetilde{E}\left(\sqrt{\rho_{1}}, \sqrt{\rho_{2}}\right)$ is strictly convex in $\rho_{1}, \rho_{2}$.

Proof. By the assumption, matrix $V$ is positive semi-definite, hence it is easy to prove that

$$
\int_{\mathbb{R}^{d}}\left[\frac{1}{2}\left(\left|\nabla \phi^{1}\right|^{2}+\left|\nabla \phi^{2}\right|^{2}\right)+|x|^{2}\left(\left|\phi^{1}\right|^{2}+\left|\phi^{2}\right|^{2}\right)+\delta\left|\phi^{1}\right|^{2}\right] d x
$$

is convex.

Next we prove the claim that

$$
\int_{\mathbb{R}^{d}}\left(v_{11}\left|\phi^{1}\right|^{2 p+2}+2 v_{12}\left|\phi^{1}\right|^{p+1}\left|\phi^{2}\right|^{p+1}+v_{22}\left|\phi^{2}\right|^{2 p+2}\right) d x
$$

is convex.

Indeed, by the condition that $v_{11} \geq 0,(p-1) v_{12} \geq 0$ and $v_{11} v_{22} \geq$ $\max \left\{\frac{1}{p}, 1\right\} v_{12}^{2}$, we can prove that $F(x, y)=v_{11} x^{p+1}+2 v_{12} x^{\frac{p+1}{2}} y^{\frac{p+1}{2}}+v_{22} y^{p+1}$ is convex for $x, y \geq 0$, and the claim is proved.

Now we need to verify the convexity of the last term, i.e.,

$$
\int_{\mathbb{R}^{d}}-\left|\phi^{1}\right| \cdot\left|\phi^{2}\right| d x
$$

Let $\sqrt{\rho_{1}}, \sqrt{\rho_{2}} \in \mathcal{D}_{1}$, and $\sqrt{\rho_{1}^{\prime}}, \sqrt{\rho_{2}^{\prime}} \in \mathcal{D}_{1}$, then for $\alpha \in(0,1)$,

$$
\sqrt{\left(\alpha \rho_{1}+(1-\alpha) \rho_{1}^{\prime}\right)}, \sqrt{\left(\alpha \rho_{2}+(1-\alpha) \rho_{2}^{\prime}\right)} \in \mathcal{D}_{1} \text {. }
$$

By Cauchy inequality, we have

$$
\alpha \sqrt{\rho_{1}} \sqrt{\rho_{1}}+(1-\alpha) \sqrt{\rho_{1}^{\prime}} \sqrt{\rho_{2}^{\prime}} \leq \sqrt{\alpha \rho_{1}+(1-\alpha) \rho_{1}^{\prime}} \times \sqrt{\alpha \rho_{2}+(1-\alpha) \rho_{2}^{\prime}} .
$$

Thus the last term is convex. This completes the proof. 
Theorem 2.3. Assume that $v_{11} \geq 0,(p-1) v_{12} \geq 0$ and $v_{11} v_{22} \geq \max \left\{\frac{1}{p}, 1\right\} v_{12}^{2}$. Then there exists a minimizer $\phi_{\infty}^{1}, \phi_{\infty}^{2} \in \mathcal{D}_{1}$ of (2.3). In addition, if at least one of the parameters $\lambda, \gamma_{1}=v_{11}-v_{22}$ and $\gamma_{2}=v_{11}-v_{12}$ is nonzero, then the minimizer $\left|\phi_{1}^{\infty}\right|,\left|\phi_{2}^{\infty}\right|$ is unique.

Proof. It is clear that $\widetilde{E}$ is bounded below by the assumption. Let $\phi_{n}^{1}, \phi_{n}^{2} \in \mathcal{D}_{1}$ be a minimizing sequence. Then there exists a constant $C$ such that $\left\|\nabla \phi_{n}^{1}\right\|+$ $\left\|\nabla \phi_{n}^{2}\right\|<C,\left\|\phi_{n}^{1}\right\|_{L^{2 p+2}}+\left\|\phi_{n}^{2}\right\|_{L^{2 p+2}}<C$ and $\int_{\mathbb{R}^{d}}\left[|x|^{2}\left|\phi_{n}^{1}\right|^{2}+|x|^{2}\left|\phi_{n}^{2}\right|^{2}\right] d x<$ $C$ for all $n \geq 0$. Therefore $\phi_{n}^{1}$ and $\phi_{n}^{2}$ belongs to a weakly compact set in $L^{2 p+2} \cdot H^{1}$ and $L_{|x|^{2}}^{2}=\left\{\left.\phi\left|\int_{\mathbb{R}^{d}}\right| x\right|^{2}\left|\phi_{n}^{1}\right|^{2} d x<\infty\right\}$ with a weighted $L^{2}$-norm given by $\|\phi\|_{L_{|x|^{2}}^{2}}=\left(\int_{\mathbb{R}^{d}}|x|^{2}|\phi|^{2} d x\right)^{1 / 2}$. Thus there exist a $\phi_{\infty}^{1}, \phi_{\infty}^{2} \in \mathcal{D}$ and a subsequence which we denote as the original sequence for simplicity, such that

$$
\begin{gathered}
\phi_{n}^{1} \rightarrow \phi_{\infty}^{1}, \quad \phi_{n}^{2} \rightarrow \phi_{\infty}^{2}, \text { in } L^{2} \cap L^{2 p+2} \cap L_{|x|^{2}}^{2} \\
\nabla \phi_{n}^{1} \rightarrow \nabla \phi_{\infty}^{1}, \quad \nabla \phi_{n}^{2} \rightarrow \nabla \phi_{\infty}^{2}, \text { in } L^{2} .
\end{gathered}
$$

Also we can suppose that $\phi_{n}^{1}$ and $\phi_{n}^{2}$ are nonnegative, since we can replace them with $\left|\phi_{n}^{1}\right|$ and $\left|\phi_{n}^{2}\right|$, which also minimizing the functionals $\widetilde{E}$. To show that $\widetilde{E}$ attains its minimal at $\phi_{\infty}^{1}, \phi_{\infty}^{2}$, we recall the constraint $\left\|\phi_{n}^{1}\right\|^{2}+\left\|\phi_{n}^{2}\right\|^{2}=1$, then the functional $\widetilde{E}$ can be rewritten as

$$
\widetilde{E}\left(\phi_{n}^{1}, \phi_{n}^{2}\right)=E_{0}\left(\phi_{n}^{1}, \phi_{n}^{2}\right)+|\lambda| \int_{\mathbb{R}^{d}}\left|\phi_{n}^{1}-\phi_{n}^{2}\right|^{2} d x-|\lambda| .
$$

First, we show that for any given $\varepsilon>0$,

$$
\int_{\mathbb{R}^{d}} v_{12}\left|\phi_{\infty}^{1}\right|^{p+1}\left|\phi_{\infty}^{2}\right|^{p+1} d x \leq \liminf _{n \rightarrow \infty} \int_{\mathbb{R}^{d}} v_{12}\left|\phi_{n}^{1}\right|^{p+1}\left|\phi_{n}^{2}\right|^{p+1} d x+\varepsilon
$$

When $v_{12} \geq 0$, this is obviously true. For $v_{12} \leq 0$, we decompose $\mathbb{R}^{d}$ into two parts, a bounded region $B_{R}=|x| \leq R$ and $B_{R}^{c}:=\mathbb{R}^{d} \backslash B$, such that $|x|^{2} \geq 1 / \eta$ on $B_{R}^{c}$, where $\eta>0$ sufficiently small. Then $\int_{B_{R}^{c}}\left(\left|\phi_{n}^{1}\right|^{2}+\left|\phi_{n}^{2}\right|^{2}\right) d x \leq C \eta$ in $B_{R}^{c}$. Using the Sobolev-Gagliardo inequality, for $d=3$ and $2^{*}=6$, we have

$$
\begin{aligned}
\int_{B_{R}^{c}}\left|\phi_{n}^{1}\right|^{2 p+2} d x & \leq \int_{B_{R}^{c}}\left|\phi_{n}^{1}\right|^{3 p} \cdot\left|\phi_{n}^{1}\right|^{2-p} d x \\
& \leq\left(\int_{B_{R}^{c}}\left|\phi_{n}^{1}\right|^{6} d x\right)^{\frac{3 p}{6}}\left(\int_{B_{R}^{c}}\left|\phi_{n}^{1}\right|^{2} d x\right)^{\frac{2-p}{2}} \\
& =\left\|\phi_{n}^{1}\right\|_{2^{*}}^{3 p}\left\|\phi_{n}^{1}\right\|_{2}^{2-p} \leq M\left\|\nabla \phi_{n}^{1}\right\|_{2}^{3 p} \cdot C \eta^{\frac{2-p}{2}} \leq M C^{3 p+1} \eta^{\frac{2-p}{2}},
\end{aligned}
$$

where $M$ is a constant. Thus, by choosing $R$ sufficiently large, we have, for all $n$,

$$
\int_{B_{R}^{c}}\left|\phi_{n}^{1}\right|^{2 p+2} d x \leq \frac{\varepsilon}{2\left(1+\left|v_{12}\right|\right)} .
$$

Similarly, by using Sobolev inequality, we can get the same result for the cases $d=1$ and $d=2$. 
The same conclusion holds for $\phi_{n}^{2}$. Notice that for $\phi_{\infty}^{1}$ and $\phi_{\infty}^{2}$, by the weak lower semicontinuous property of $L^{2 p+2}\left(\mathbb{R}^{d}\right)$-norm, $H^{1}\left(\mathbb{R}^{d}\right)$-norm and $L_{|x|^{2}}^{2}\left(\mathbb{R}^{d}\right)$-norm, we can have

$$
\begin{aligned}
& \left\|\nabla \phi_{\infty}^{1}\right\|+\left\|\nabla \phi_{\infty}^{2}\right\|<C,\left\|\phi_{\infty}^{1}\right\|_{L^{2 p+2}}+\left\|\phi_{\infty}^{2}\right\|_{L^{2 p+2}}<C \text { and } \\
& \int_{\mathbb{R}^{d}}\left[|x|^{2}\left|\phi_{\infty}^{1}\right|^{2}+|x|^{2}\left|\phi_{\infty}^{2}\right|^{2}\right] d x<C .
\end{aligned}
$$

Following the above arguments, the same conclusion holds for $\phi_{\infty}^{1}$ and $\phi_{\infty}^{2}$, i.e., we have

$$
\begin{aligned}
& \int_{B_{R}^{c}}\left|\phi_{n}^{j}\right|^{2 p+2} d x \leq \frac{\varepsilon}{2\left(1+\left|v_{12}\right|\right)}, \\
& \int_{B_{R}^{c}}\left|\phi_{\infty}^{j}\right|^{2 p+2} d x \leq \frac{\varepsilon}{2\left(1+\left|v_{12}\right|\right)}, j=1,2, N \geq 0 .
\end{aligned}
$$

Then, by the Cauchy-Schwarz inequality, we have for $n \geq 0$

$$
\begin{aligned}
\left.\left|\int_{B_{R}^{c}} v_{12}\right| \phi_{n}^{1}\right|^{p+1}\left|\phi_{n}^{2}\right|^{p+1} d x \mid & \leq\left|v_{12}\right|\left(\int_{B_{R}^{c}}\left|\phi_{n}^{1}\right|^{2 p+2} \mid d x\right)^{\frac{1}{2}}\left(\int_{B_{R}^{c}}\left|\phi_{n}^{2}\right|^{2 p+2} \mid d x\right)^{\frac{1}{2}} \\
& \leq \frac{\varepsilon}{2},
\end{aligned}
$$

and

$$
\left.\left|\int_{B_{R}^{c}} v_{12}\right| \phi_{\infty}^{1}\right|^{p+1}\left|\phi_{\infty}^{2}\right|^{p+1} d x \mid \leq \frac{\varepsilon}{2} .
$$

Next, in the ball $B_{R}$, applying the Sobolev embedding theorem, the strong convergence holds,

$$
\phi_{n}^{1} \rightarrow \phi_{\infty}^{1}, \quad \phi_{n}^{2} \rightarrow \phi_{\infty}^{2}, \text { in } L^{2}\left(B_{R}\right) \cap L^{2 p+2}\left(B_{R}\right) .
$$

By writing

$$
\begin{gathered}
\left.\quad\left|\int_{B_{R}} v_{12}\right| \phi_{n}^{1}\right|^{p+1}\left|\phi_{n}^{2}\right|^{p+1} d x-\int_{B_{R}} v_{12}\left|\phi_{\infty}^{1}\right|^{p+1}\left|\phi_{\infty}^{2}\right|^{p+1} d x \mid \\
\leq\left|v_{12}\right|\left(\mid \int_{B_{R}}\left(\left.\left.\left|\phi_{n}^{1}\right|^{p+1}|-| \phi_{\infty}^{1}\right|^{p+1}|| \phi_{n}^{2}\right|^{p+1}|d x|\right.\right. \\
\quad+\mid \int_{B_{R}}\left(\left.\left.\left|\phi_{n}^{2}\right|^{p+1}|-| \phi_{\infty}^{2}\right|^{p+1}|| \phi_{\infty}^{2}\right|^{p+1}|d x|\right) \\
\leq C\left(\left\|\phi_{n}^{1}-\phi_{\infty}^{1}\right\|_{L^{2 p+2}\left(B_{R}\right)}+\left\|\phi_{n}^{2}-\phi_{\infty}^{2}\right\|_{L^{2 p+2}\left(B_{R}\right)}\right) .
\end{gathered}
$$

Hence, the inequality (2.4) holds by combining the above results.

In a similar argument, we can prove that

$$
\limsup _{n \rightarrow \infty}\left|\int_{\mathbb{R}^{d}}\left(\left|\phi_{n}^{1}\right|^{p+1}+\left|\phi_{n}^{2}\right|^{p+1}\right) d x-\int_{\mathbb{R}^{d}}\left(\left|\phi_{\infty}^{1}\right|^{p+1}+\left|\phi_{\infty}^{2}\right|^{p+1}\right) d x\right| \leq \varepsilon .
$$


Since $L^{2 p+2}\left(\mathbb{R}^{d}\right)$-norm, $H^{1}\left(\mathbb{R}^{d}\right)$-norm and $L_{|x|^{2}}^{2}\left(\mathbb{R}^{d}\right)$-norm, are all weakly lower semicontinuous, we have

$$
\widetilde{E}\left(\phi_{\infty}^{1}, \phi_{\infty}^{2}\right) \leq \liminf _{n \rightarrow \infty} \widetilde{E}\left(\phi_{n}^{1}, \phi_{n}^{2}\right)+\varepsilon, \varepsilon \geq 0
$$

which immediately implies that $\widetilde{E}\left(\phi_{\infty}^{1}, \phi_{\infty}^{2}\right) \leq \liminf _{n \rightarrow \infty} \widetilde{E}\left(\phi_{n}^{1}, \phi_{n}^{2}\right)$. Moreover, $\phi_{\infty}^{1}, \phi_{\infty}^{2} \in \mathcal{D}_{1}$, by (2.5) which implies the existence of minimizer of the problem $(2.3)$.

In addition, if at least one of the parameters $\lambda, \gamma_{1}, \gamma_{2}$ is nonzero, the uniqueness of $\left|\phi_{\infty}^{1}\right|,\left|\phi_{\infty}^{2}\right|$ follows from the strict convexity of $\widetilde{E}$. For the case $\delta \neq 0$ and $\lambda=\gamma_{1}=\gamma_{2}=0$, the uniqueness is easy to derive.

Combining the results in Lemma 2.1 and Theorem 2.1, we immediately have the following existence and uniqueness results for the ground states of (1.3):

Theorem 2.4. Assume that $v_{11} \geq 0,(p-1) v_{12} \geq 0$ and $v_{11} v_{22} \geq \max \left\{\frac{1}{p}, 1\right\} v_{12}^{2}$. Then there exists a ground state $\phi_{g}^{1}, \phi_{g}^{2}$ of (1.3). Furthermore, $e^{i \theta_{1}}\left|\phi_{g}^{1}\right|, e^{i \theta_{2}}\left|\phi_{g}^{2}\right|$ is also a ground state of (1.3) with $\theta_{1}$ and $\theta_{2}$ two constants satisfying $\theta_{1}-\theta_{2}=$ $\pm \pi$ when $\lambda>0$ and $\theta_{1}=\theta_{2}$ when $\lambda<$, respectively. In addition, if at least one of the parameters $\delta, \lambda, \gamma_{1}=v_{11}-v_{22}$ and $\gamma_{2}=v_{11}-v_{12}$ is nonzero, then the ground state $\left|\phi_{1}^{g}\right|,-\operatorname{sign}(\lambda)\left|\phi_{2}^{g}\right|$ is unique. In contrast, if $\frac{2}{3}<p<\frac{2}{d-2}$, $d=2,3$, and $v_{11}<0$ or $v_{22}<0$ or $v_{12}<0$ with $v_{12}^{2}>v_{11} v_{22}$, then there exist no ground states of (1.3).

Proof. The first part of the theorem follows from Lemma 2.1 and Theorem 2.3. We are going to prove the nonexistence results.

In the case where $d=3$, choose $\phi_{\varepsilon}^{1}=\frac{\sqrt{\theta}}{(\varepsilon \pi)^{3 / 4}} e^{-\frac{|x|^{2}}{2 \varepsilon}}$, and $\phi_{\varepsilon}^{2}=\frac{\sqrt{1-\theta}}{(\varepsilon \pi)^{3 / 4}} e^{-\frac{|x|^{2}}{2 \varepsilon}}$, $\theta \in[0,1], \varepsilon>0$.

When $v_{11}<0$, choosing $\theta=1$, i.e., $\phi_{\varepsilon}^{1}=\frac{1}{(\varepsilon \pi)^{3 / 4}} e^{-\frac{|x|^{2}}{2 \varepsilon}}, \phi_{\varepsilon}^{2}=0$, we obtain

$$
\begin{gathered}
\int_{\mathbb{R}^{d}} \frac{1}{2}\left(\left|\nabla \phi_{\varepsilon}^{1}\right|^{2}+\left|\nabla \phi_{\varepsilon}^{2}\right|^{2}\right) d x=\frac{3}{4} \varepsilon^{-1}, \\
\int_{\mathbb{R}^{d}}|x|^{2}\left(\left|\phi_{\varepsilon}^{1}\right|^{2}+\left|\phi_{\varepsilon}^{2}\right|^{2}\right) d x=\frac{3}{2} \varepsilon \\
\int_{\mathbb{R}^{d}} \delta\left|\phi_{\varepsilon}^{1}\right|^{2} d x=\delta
\end{gathered}
$$

and

$$
\begin{aligned}
& \frac{1}{2 p+2} \int_{\mathbb{R}^{n}}\left(v_{11}\left|\phi_{\varepsilon}^{1}\right|^{2 p+2}+v_{22}\left|\phi_{\varepsilon}^{2}\right|^{2 p+2}+2 v_{12}\left|\phi_{\varepsilon}^{1}\right|^{p+1}\left|\phi_{\varepsilon}^{2}\right|^{p+1}\right) d x \\
= & \frac{v_{11}}{2}(p+1)^{-\frac{5}{2}}(\pi \varepsilon)^{-\frac{3 p}{2}} .
\end{aligned}
$$

Hence, we have

$$
E\left(\phi_{\varepsilon}^{1}, 0\right)=\frac{3}{4} \varepsilon^{-1}+\frac{3}{2} \varepsilon+1+\frac{v_{11}}{2}(p+1)^{-\frac{5}{2}}(\pi \varepsilon)^{-\frac{3 p}{2}} .
$$


Then by $p>\frac{2}{3}, \lim _{\varepsilon \rightarrow 0^{+}} E\left(\phi_{\varepsilon}^{1}, 0\right)=-\infty$.

When $v_{22}<0$, choosing $\theta=0$, i.e., $\phi_{\varepsilon}^{2}=\frac{1}{(\varepsilon \pi)^{3 / 4}} e^{-\frac{|x|^{2}}{2 \varepsilon}}, \phi_{\varepsilon}^{1}=0$, we obtain

$$
E\left(0, \phi_{\varepsilon}^{2}\right)=\frac{3}{4} \varepsilon^{-1}+\frac{3}{2} \varepsilon+\frac{v_{22}}{2}(p+1)^{-\frac{5}{2}}(\pi \varepsilon)^{-\frac{3 p}{2}} .
$$

Then by $p>\frac{2}{3}, \lim _{\varepsilon \rightarrow 0^{+}} E\left(0, \phi_{\varepsilon}^{2}\right)=-\infty$.

When $v_{11} \geq 0, v_{22} \geq 0, v_{12}<0$ and $v_{12}^{2}>v_{11} v_{22}$, choosing

$$
\theta=\frac{\left(v_{22}-v_{12}\right)^{\frac{2}{p+1}}}{\left(v_{11}-v_{12}\right)^{\frac{2}{p+1}}+\left(v_{22}-v_{12}\right)^{\frac{2}{p+1}}} \in(0,1)
$$

then

$$
\begin{aligned}
v_{\theta}: & =v_{11} \theta^{p+1}+2 v_{12} \theta^{\frac{p+1}{2}}(1-\theta)^{\frac{p+1}{2}}+v_{22}(1-\theta)^{p+1} \\
& =\frac{\left(v_{11} v_{22}-v_{12}^{2}\right)\left(v_{11}+v_{22}-2 v_{12}\right)}{\left(\left(v_{11}-v_{12}\right)^{\frac{2}{p+1}}+\left(v_{22}-v_{12}\right)^{\frac{2}{p+1}}\right)^{p+1}}<0,
\end{aligned}
$$

and

$$
E\left(\psi_{\varepsilon}^{1}, \psi_{\varepsilon}^{2}\right)=\frac{3}{4} \varepsilon^{-1}+\frac{3}{2} \varepsilon+\delta \theta+\frac{1}{2}(p+1)^{-\frac{5}{2}}(\pi \varepsilon)^{-\frac{3 p}{2}} v_{\theta}+2 \lambda \sqrt{\theta(1-\theta)} .
$$

Then, by $p>\frac{2}{3}, \lim _{\varepsilon \rightarrow 0^{+}} E\left(\phi_{\varepsilon}^{1}, \phi_{\varepsilon}^{2}\right)=-\infty$. Thus, there exists no ground state in these cases.

In the case $d=2$, similar to the above method, we can obtain the same conclusion holds. This completes the proof.

\section{Properties of the ground states}

In this section, we will show some properties of the ground states with large parameters $\lambda$ or $\delta$.

Let us define

$$
\begin{gathered}
E_{1}(\phi)=\int_{\mathbb{R}^{d}}\left[\frac{1}{2}|\nabla \phi|^{2}+\left(|x|^{2}+\frac{\delta}{2}\right)|\phi|^{2}+\frac{v_{11}+2 v_{12}+v_{22}}{2}|\phi|^{2 p+2}\right], \\
E_{2}(\phi)=\int_{\mathbb{R}^{d}}\left[\frac{1}{2}|\nabla \phi|^{2}+|x|^{2}|\phi|^{2}+\frac{\beta}{2}|\phi|^{2 p+2}\right] .
\end{gathered}
$$

Similarly to the argument as in [6], it is easy to prove that there exists a unique positive minimizer of (3.1) under the constraint

$$
\|\phi\|_{L^{2}}^{2}=\int_{\mathbb{R}^{d}}|\phi|^{2} d x=\frac{1}{2},
$$

and there exists a unique positive minimizer of (3.2) under the constraint

$$
\|\phi\|_{L^{2}}^{2}=\int_{\mathbb{R}^{d}}|\phi|^{2} d x=1 .
$$


Theorem 3.1. Suppose $v_{11} \geq 0,(p-1) v_{12} \geq 0$ and $v_{11} v_{22} \geq \max \left\{\frac{1}{p}, 1\right\} v_{12}^{2}$. For fixed $\delta$, there exists $\lambda_{0}>0$ such that, for every $\lambda>\lambda_{0}$, ground state $\phi_{\lambda}^{1}, \phi_{\lambda}^{2}$ of (1.3) with respect to $\lambda$, satisfying $\phi_{\lambda}^{1} \neq 0, \phi_{\lambda}^{2} \neq 0$.

Proof. Without loss of generality, we assume $\lambda<0$ and the ground state $\phi_{\lambda}^{1} \geq$ $0, \phi_{\lambda}^{2} \geq 0$. Since $\phi_{\lambda}^{1}, \phi_{\lambda}^{2} \in \mathcal{D}_{1}$, we have

$$
\widetilde{E}\left(\left|\psi_{\lambda}^{1}\right|,\left|\psi_{\lambda}^{2}\right|\right) \leq \widetilde{E}\left(\psi_{g}, \psi_{g}\right)
$$

where $\phi_{g}$ is the unique positive minimizer of (3.1) under the constraint (3.3).

$$
\text { Noticing }
$$

$$
\widetilde{E}\left(\psi^{1}, \psi^{2}\right)=E_{0}\left(\psi_{1}, \psi_{2}\right)+|\lambda| \int_{\mathbb{R}^{d}}\left|\phi^{1}-\phi^{2}\right|^{2} d x-|\lambda|, \phi^{1}, \phi^{2} \in \mathcal{D}_{1},
$$

we have

$$
\widetilde{E}\left(\psi_{g}, \psi_{g}\right)=2 E_{1}\left(\psi_{g}\right)-|\lambda| .
$$

Substituting (3.7) into (3.5) and noticing (3.6), there exists a constant $C>0$ such that

$$
\left\|\phi_{\lambda}^{1}-\phi_{\lambda}^{2}\right\|_{L^{2}} \leq \frac{C}{|\lambda|},|\lambda|>0 .
$$

Then the fact $\phi_{\lambda}^{1}, \phi_{\lambda}^{2} \in \mathcal{D}_{1}$ and (3.8) imply the conclusion.

Theorem 3.2. Suppose $v_{11} \geq 0,(p-1) v_{12} \geq 0$ and $v_{11} v_{22} \geq \max \left\{\frac{1}{p}, 1\right\} v_{12}^{2}$. For fixed $\lambda$, there exists $\delta_{0}>0$ such that, for every $|\delta|>\delta_{0}$, there exists $\varepsilon_{0}>0$ ground state $\phi_{\delta}^{1}, \phi_{\delta}^{2}$ of (1.3) with respect to $\delta$, satisfying $\left|\left\|\phi_{\delta}^{1}\right\|_{L^{2}}-\left\|\phi_{\delta}^{2}\right\|_{L^{2}}\right|>$ $1-\varepsilon_{0}$.

Proof. When $\delta>0$, we take $\beta=v_{22}$ in (3.2). Since $0, \phi_{g} \in \mathcal{D}_{1}$, we have

$$
\widetilde{E}\left(\left|\psi_{\delta}^{1}\right|,\left|\psi_{\delta}^{2}\right|\right) \leq \widetilde{E}\left(0, \psi_{g}\right),
$$

where $\phi_{g}$ is the unique positive minimizer of (3.2) under the constraint (3.4). Noticing

$$
\widetilde{E}\left(\psi^{1}, \psi^{2}\right)=E_{0}\left(\psi_{1}, \psi_{2}\right)+|\lambda| \int_{\mathbb{R}^{d}}\left|\phi^{1}-\phi^{2}\right|^{2} d x-|\lambda|, \phi^{1}, \phi^{2} \in \mathcal{D}_{1},
$$

we have

$$
\widetilde{E}\left(0, \psi_{g}\right)=E_{2}\left(\psi_{g}\right)+|\lambda| \int_{\mathbb{R}^{d}}|\phi-g|^{2} d x-|\lambda| .
$$

Substituting (3.11) into (3.9) and noticing (3.10), there exists a constant $C>0$ such that

$$
\left\|\phi_{\delta}^{1}\right\|_{L^{2}}^{2} \leq \frac{C}{\delta} .
$$

Furthermore the fact $\phi_{\delta}^{1}, \phi_{\delta}^{2} \in \mathcal{D}_{1}$ and (3.12) imply

$$
\left\|\phi_{\delta}^{2}\right\|_{L^{2}}^{2}>1-\frac{C}{\delta}
$$


Then there exists $\delta_{0}>0$, for any $\delta>\delta_{0}$, there exists $\varepsilon_{0}>0$ such that

$$
\left\|\phi_{\delta}^{1}\right\|_{L^{2}}<\frac{\varepsilon_{0}}{2}
$$

and

$$
\left\|\phi_{\delta}^{2}\right\|_{L^{2}}>1-\frac{\varepsilon_{0}}{2}
$$

(3.14) and (3.15) imply the conclusion.

When $\delta<0$, we take $\beta=v_{11}$ in (3.2). Using the fact $\phi_{g}, 0 \in \mathcal{D}_{1}$, the conclusion can be established by similar argument as the case $\delta>0$.

Acknowledgements. The authors would like to thank for the valuable suggestions of the referee.

\section{References}

[1] W. Bao, Ground states and dynamics of multicomponent Bose-Einstein condensates, Multiscale Model. Simul. 2 (2004), no. 2, 210-236.

[2] W. Bao and Y. Cai, Ground states of two-component Bose-Einstein condensates with an internal atomic Josephson junction, East Asia Journal of Applied Mathematics 1 (2011), no. 1, 49-81.

[3] S. M. Chang, C. S. Lin, T. C. Lin, and W. W. Lin, Segregated nodal domains of twodimensional multispecies Bose-Einstein condensates, Phys. D 196 (2004), no. 3-4, 341361.

[4] S. M. Chang, W. W. Lin, and S. F. Shieh, Gauss-Seidel-type methods for energy states of a multi-component Bose-Einstein condensate, J. Comput. Phys. 202 (2005), no. 1, 367-390.

[5] D. Jaksch, S. A. Gardiner, K. Schulze, J. I. Cirac, and P. Zoller, Uniting Bose-Einstein condensate in optical resonators, Phys. Rev. Lett. 86 (2001), no. 21, 4733-4736.

[6] E. H. Lieb, R. Seiringer, and J. Yngvason, Bosons in a trap: a rigorous derivation of the Gross-Pitaevskii energy functional, Phy. Rev. A 61 (2000), article 043602.

[7] E. H. Lieb and J. P. Solovej, Ground state energy of the two-component charged Bose gas, Comm. Math. Phys. 252 (2004), no. 1-3, 485-534.

[8] L. P. Pitaevskii and S. Stringari, Bose-Einstein Condensation, Clarendon Press, 2003.

[9] J. Williams, R. Walser, J. Cooper, E. Cornell, and M. Holland, Nonlinear Josephsontype oscillations of a driven two-component Bose-Einstein condensate, Phys. Rev. A 59 (1999), article R31-R34.

[10] Y. Zhang, W. Bao, and H. Li, Dynamics of rotating two-component Bose-Einstein condensates and its efficient computation, Phys. D 234 (2007), no. 1, 49-69.

\section{ZhongXue Lü}

School of Mathematical Sciences

JiangSu Normal University

Xuzhou, 221116, P. R. China

E-mail address: 1vzx1@tom.com

ZUHAN LIU

YANGZHOU UNIVERSITY

YAngzhou, 225002, P. R. China

E-mail address: zuhanl@yahoo.com 